\title{
The scaled Hermite-Weber basis in the spectral and pseudospectral pictures
}

\author{
H. Taşeli* and H. Alıcı \\ Department of Mathematics, Middle East Technical University, 06531 Ankara, Turkey \\ E-mail: taseli@metu.edu.tr
}

Received 13 January 2005

\begin{abstract}
Computational efficiencies of the discrete (pseudospectral, collocation) and continuous (spectral, Rayleigh-Ritz, Galerkin) variable representations of the scaled HermiteWeber basis in finding the energy eigenvalues of Schrödinger operators with several potential functions have been compared. It is well known that the so-called differentiation matrices are neither skew-symmetric nor symmetric in a pseudospectral formulation of a differential equation, unlike their Rayleigh-Ritz counterparts. In spite of this fact, it is shown here that the spectra of matrix Hamiltonians generated by Hermite collocation method may be determined by way of diagonalizing symmetric matrices. Furthermore, the symmetric matrix elements do not require the evaluation of Hermite polynomials at the grid points. Surprisingly, the present numerical results suggest that the convergence rates of collocation and Rayleigh-Ritz methods are entirely the same.
\end{abstract}

KEY WORDS: Schrödinger operator, quantum mechanical oscillators, singular SturmLiouville problems on the real line, spectral and pseudospectral methods, HermiteWeber functions, Hermite collocation points

AMS subject classification: 65L60, 81Q05, 65L15, 34L40, 42C10

\section{Introduction}

Recently, Taşeli and Erseçen [1] (hereafter referred to as PI) presented an extensive numerical analysis of the singular Sturm-Liouville eigenvalue problems described by one-dimensional Hamiltonians

$$
\mathcal{H}=-\frac{\mathrm{d}^{2}}{\mathrm{~d} x^{2}}+V(x), \quad x \in(-\infty, \infty)
$$

for a variety of quantum mechanical potentials $V(x)$. In PI and many others $[2,3]$, this problem is considered with a scaled variable $\xi$,

$$
\xi=\alpha x, \quad \alpha>0,
$$

${ }^{*}$ Corresponding author. 
which transforms the Schrödinger equation $\mathcal{H} \Psi=E \Psi$ into the form

$$
\left[-\frac{\mathrm{d}^{2}}{\mathrm{~d} \xi^{2}}+\alpha^{-2} V\left(\alpha^{-1} \xi\right)\right] \Psi(\xi)=\mathcal{E}(\alpha) \Psi(\xi), \quad \Psi \in L_{2}(-\infty, \infty),
$$

whose eigenfunctions $\Psi$ should be in the Hilbert space $L_{2}$ of square integrable functions. Clearly, the eigenvalues $\mathcal{E}(\alpha)$ of (3) are connected with the eigenvalues $E$ of the original system by the formula

$$
E=\alpha^{2} \mathcal{E}(\alpha)
$$

depending on the optimization parameter $\alpha$.

Generally speaking, in a Galerkin spectral method, the solution of a differential equation is expanded into a series of the form

$$
\Psi(\xi)=\lim _{N \rightarrow \infty} \sum_{n=0}^{N} c_{n} \phi_{n}(\xi),
$$

where the sequence $\left\{\phi_{n}(\xi)\right\}$ denotes a prescribed complete and orthogonal basis of the space to which $\Psi(\xi)$ belongs. In practice, the integer index $N$ is taken as sufficiently large but a finite number, standing for the order of approximation. In this approach, $\Psi(\xi)$ is characterized continuously in $\xi$ by the spectral or Fourier coefficients $c_{n}$, and once they have been found, the solution can immediately be approximated at an arbitrary point $\xi$ in the domain of interest. On the other hand, in a pseudospectral method, the solution is proposed to be a function of the same form $\Psi(\xi)$ of (5), but this time it is represented by its actual values $\Psi\left(\xi_{m}\right)$ at $N+1$ discrete collocation or grid points $\xi_{0}, \xi_{1}, \ldots, \xi_{N}$. Hence the truncated version of (5) may now be interpreted philosophically as a linear interpolation formula, which enables us to determine the solution $\Psi(\xi)$, of course, approximately for any desired value of $\xi$ other than the collocation points $\left\{\xi_{m}\right\}$.

As a terminology, more general Galerkin spectral method is conventionally called Rayleigh-Ritz method when a self-adjoint differential operator like $\mathcal{H}$ is under consideration. The numerical performance of the Rayleigh-Ritz spectral method based on the normalized Hermite-Weber functions

$$
\phi_{n}(\xi)=\frac{1}{\sqrt{2^{n} n ! \sqrt{\pi}}} \mathrm{e}^{-\xi^{2} / 2} H_{n}(\xi), \quad n=0,1, \ldots
$$

wherein the $H_{n}(\xi)$ are the classical Hermite polynomials, has been tested in PI in the treatment of the eigenvalue problem (3) for symmetric and asymmetric polynomial potentials with both single and double wells, as well as for nonpolynomial Gaussian and Morse type oscillators. Thus the main objective of this article is to investigate the numerical implementation of the same orthonormal basis set $\left\{\phi_{n}(\xi)\right\}$ in a pseudospectral picture. 
Keeping in mind the interdisciplinary character of the Journal of Mathematical Chemistry, in section 2 we review the foundations of pseudospectral techniques to help the readers from other areas of expertise. Section 3 is concerned with the matrix elements developing for the application of the Hermite pseudospectral method (HPM) to the aforementioned Schrödinger equation in (3). The last section contains the numerical results and concluding remarks.

\section{Polynomial interpolation and differentiation matrices}

The basic idea behind a pseudospectral method is the polynomial interpolation, which is one of the oldest, and perhaps most applied, problems in mathematics. The aim of this section is to remind briefly the concept of constructing a polynomial approximation to a continuously differentiable function $f(\xi)$, which passes through a number of support points $\left(\xi_{n}, f_{n}\right)$. To be more specific, we assume that the values $f_{n}=f\left(\xi_{n}\right)$ of the function are known, at least, at the nodes or grid points $\xi_{0}, \xi_{1}, \ldots, \xi_{N}$, the number of which is conveniently taken as $N+1$ with a positive integer $N \geqslant 1$.

Now let us consider a class of $N$ th degree polynomials of the form

$$
\ell_{n}(\xi)=\frac{\pi(\xi)}{\left(\xi-\xi_{n}\right) \pi^{\prime}\left(\xi_{n}\right)}, \quad n=0,1, \ldots
$$

where

$$
\pi(\xi)=\kappa \prod_{m=0}^{N}\left(\xi-\xi_{m}\right)
$$

stands for a polynomial of degree $N+1$ having the real and distinct roots $\xi_{0}<$ $\xi_{1}<\cdots<\xi_{N}$ that are located at the nodes. The constant $\kappa$ is theoretically redundant and may be taken as unity to have a monic polynomial. Alternatively, it can be regarded as a free flexible parameter for a standardization of another kind, which plays a remarkable role in a numerical algorithm. The set $\left\{\ell_{n}(\xi)\right\}$ of $N$ th degree polynomials comprises the celebrated Lagrange polynomials [4] with the property that

$$
\ell_{n}\left(\xi_{m}\right)=\delta_{m n},
$$

where $\delta_{m n}$ is the Kronecker's delta. In what follows, any function $f(\xi)$ for which $f_{n}=f\left(\xi_{n}\right)$ has a unique $N$ th degree polynomial approximation $p(\xi)$ of the form

$$
p(\xi)=\sum_{n=0}^{N} \ell_{n}(\xi) f_{n}, \quad f(\xi)=p(\xi)+e_{N}(\xi)
$$


known as the Lagrange interpolation formula, satisfying

$$
p\left(\xi_{n}\right)=f_{n}, \quad n=0,1, \ldots, N
$$

so that the error term $e_{N}(\xi)$ in (10) vanishes at the nodes $\xi=\xi_{n}$. More on the interpolation error or the remainder $e_{N}(\xi)$ can be found by perusing the literature (see for example [5]). Here, we have to deduce that such an interpolation polynomial $p(\xi)$ has been defined as that polynomial of the lowest degree, $N$ in this particular instance, which agrees with the approximated function $f(\xi)$ at a specified set of $N+1$ grid points. As another important remark, if $f(\xi)$ itself were a polynomial of degree $N$ then $p(\xi)$ would be merely an alternative exact representation of that polynomial.

We may also approximate the derivative function $f^{\prime}(\xi)$ by making use of the derivative $p^{\prime}(\xi)$,

$$
p^{\prime}(\xi)=\sum_{n=0}^{N} \ell_{n}^{\prime}(\xi) f_{n}, \quad f^{\prime}(\xi)=p^{\prime}(\xi)+e_{N}^{\prime}(\xi)
$$

of the polynomial interpolant $p(\xi)$ in (10). Furthermore, the derivative values at the same $N+1$ nodes $\left\{\xi_{n}\right\}$ can be determined in terms of $f_{n}=p\left(\xi_{n}\right)$ by means of a differentiation matrix defined by

$$
\boldsymbol{D}^{(1)}:=\left[d_{m n}^{(1)}\right]=\left[\ell_{n}^{\prime}\left(\xi_{m}\right)\right]
$$

for $m, n=0,1, \ldots, N$. Actually, we face up the system

$$
p^{\prime}\left(\xi_{m}\right)=\sum_{n=0}^{N} \ell_{n}^{\prime}\left(\xi_{m}\right) f_{n}=\sum_{n=0}^{N} d_{m n}^{(1)} p\left(\xi_{n}\right), \quad m=0,1, \ldots, N,
$$

which can be written in matrix-vector form as

$$
\boldsymbol{f}^{(1)}=\boldsymbol{D}^{(1)} \boldsymbol{f}
$$

where $\boldsymbol{f}^{(1)}=\left[p^{\prime}\left(\xi_{0}\right), p^{\prime}\left(\xi_{1}\right), \ldots, p^{\prime}\left(\xi_{N}\right)\right]^{\mathrm{T}}$ and $\boldsymbol{f}=\left[p\left(\xi_{0}\right), p\left(\xi_{1}\right), \ldots, p\left(\xi_{N}\right)\right]^{\mathrm{T}}$. It should be noted that, unless otherwise stated, each matrix in this work denotes a square matrix of size $(N+1) \times(N+1)$, and all vectors are from $\mathbb{R}^{N+1}$.

Explicit expressions of the entries $d_{m n}^{(1)}$ of the first order differentiation matrix $\boldsymbol{D}^{(1)}$ follow directly from the definition of the Lagrange polynomials in (7). Therefore, it is not difficult to see that

$$
d_{m n}^{(1)}=\frac{1}{2} \begin{cases}\frac{2 \pi^{\prime}\left(\xi_{m}\right)}{\left(\xi_{m}-\xi_{n}\right) \pi^{\prime}\left(\xi_{n}\right)} & \text { if } m \neq n \\ \frac{\pi^{\prime \prime}\left(\xi_{n}\right)}{\pi^{\prime}\left(\xi_{n}\right)} & \text { if } m=n\end{cases}
$$

where $m, n=0,1, \ldots, N$. 
In a similar fashion, the second derivative values $p^{\prime \prime}\left(\xi_{m}\right)$ at the grid points may be calculated by a matrix-vector product of form (15)

$$
\boldsymbol{f}^{(2)}=\boldsymbol{D}^{(2)} \boldsymbol{f}
$$

in which $\boldsymbol{f}^{(2)}=\left[p^{\prime \prime}\left(\xi_{0}\right), p^{\prime \prime}\left(\xi_{1}\right), \ldots, p^{\prime \prime}\left(\xi_{N}\right)\right]^{\mathrm{T}}$ and $\boldsymbol{D}^{(2)}:=\left[d_{m n}^{(2)}\right]$ with

$$
d_{m n}^{(2)}=\ell_{n}^{\prime \prime}\left(\xi_{m}\right)
$$

leading to the result

$$
d_{m n}^{(2)}=\frac{1}{3} \begin{cases}\frac{3}{\xi_{m}-\xi_{n}}\left[\frac{\pi^{\prime \prime}\left(\xi_{m}\right)}{\pi^{\prime}\left(\xi_{n}\right)}-2 d_{m n}^{(1)}\right] & \text { if } m \neq n, \\ \frac{\pi^{\prime \prime \prime}\left(\xi_{n}\right)}{\pi^{\prime}\left(\xi_{n}\right)} & \text { if } m=n\end{cases}
$$

for the elements of the second order differentiation matrix $\boldsymbol{D}^{(2)}$. It is obvious that the introduction of differentiation matrices up to second order suffices for a numerical treatment of a second order differential operator. A simple method for the generation of higher order pseudospectral matrices was carried out by Welfert [6].

\section{Matrix representation of the Schrödinger operator in HPM}

By setting

$$
\Psi(\xi)=\mathrm{e}^{-\xi^{2} / 2} y(\xi),
$$

it is more preferable to factor off the weight $e^{-\xi^{2} / 2}$ in (6) and to rewrite the Schrödinger equation (3) as

$$
-y^{\prime \prime}+2 \xi y^{\prime}+[q(\xi)+1] y=\mathcal{E}(\alpha) y
$$

with the new dependent variable $y(\xi)$, where

$$
q(\xi)=\alpha^{-2} V\left(\alpha^{-1} \xi\right)-\xi^{2}
$$

denotes the modified potential.

With (20), the Rayleigh-Ritz trial function used in our earlier paper PI reads as

$$
y_{\mathrm{T}}(\xi)=\sum_{n=0}^{N} c_{n} \psi_{n}(\xi),
$$

where the $\psi_{n}(\xi)$ stand for the normalized Hermite polynomials

$$
\psi_{n}(\xi)=\frac{1}{\sqrt{2^{n} n ! \sqrt{\pi}}} H_{n}(\xi), \quad n=0,1, \ldots
$$


satisfying the Hermite differential equation $y^{\prime \prime}-2 \xi y^{\prime}+2 n y=0$. However, the usual three-term recursion $H_{n+1}=2 \xi H_{n}-2 n H_{n-1}$ of the $H_{n}$ takes the form

$$
\sqrt{n} \psi_{n-1}(\xi)-\sqrt{2} \xi \psi_{n}(\xi)+\sqrt{n+1} \psi_{n+1}(\xi)=0, \quad n=0,1, \ldots, N, \ldots
$$

while the differential-difference relation $H_{n}^{\prime}=2 n H_{n-1}$ becomes

$$
\psi_{n}^{\prime}(\xi)=\sqrt{2 n} \psi_{n-1}(\xi), \quad n=1,2, \ldots,
$$

where $\psi_{-1}(\xi)=0$ and $\psi_{0}(\xi)=\pi^{-1 / 4}$.

In this work, instead of the $N$ th degree polynomial trial solution in (23), we may deal with its formal exact representation

$$
y_{\mathrm{T}}(\xi)=\sum_{n=0}^{N} y_{n} \ell_{n}(\xi)
$$

suggested by the Lagrange interpolation formula in (10), where

$$
\ell_{n}(\xi)=\frac{\psi_{N+1}(\xi)}{\left(\xi-\xi_{n}\right) \psi_{N+1}^{\prime}\left(\xi_{n}\right)}=\frac{H_{N+1}(\xi)}{\left(\xi-\xi_{n}\right) H_{N+1}^{\prime}\left(\xi_{n}\right)}, \quad n=0,1, \ldots, N
$$

are the Lagrange polynomials appropriate to the specific problem. Thus we have assigned the collocation points as the real and distinct roots of $\psi_{N+1}(\xi)$ or $H_{N+1}(\xi)$, i.e.

$$
\pi(\xi)=\psi_{N+1}(\xi) \text { and } \quad \psi_{N+1}\left(\xi_{m}\right)=0
$$

in accordance with (8). It is interesting to notice that the first $N+1$ equations in (25) may be written as an inhomogeneous linear system

$$
\left[\begin{array}{ccccc}
-\sqrt{2} \xi & \sqrt{1} & 0 & \cdots & 0 \\
\sqrt{1} & -\sqrt{2} \xi & \sqrt{2} & \ddots & \vdots \\
0 & \sqrt{2} & \ddots & \ddots & 0 \\
\vdots & \ddots & \ddots & -\sqrt{2} \xi & \sqrt{N} \\
0 & \cdots & 0 & \sqrt{N} & -\sqrt{2} \xi
\end{array}\right]\left[\begin{array}{c}
\psi_{0}(\xi) \\
\psi_{1}(\xi) \\
\vdots \\
\psi_{N-1}(\xi) \\
\psi_{N}(\xi)
\end{array}\right]=\left[\begin{array}{c}
0 \\
0 \\
\vdots \\
0 \\
r(\xi)
\end{array}\right]
$$

with $r(\xi)=-\sqrt{N+1} \psi_{N+1}(\xi)$, which implies a homogeneous one when $\psi_{N+1}(\xi)=0$. In this case, we encounter the standard matrix eigenvalue problem $\boldsymbol{R} \boldsymbol{x}=\mu \boldsymbol{x}$, where $\boldsymbol{R}$ is a symmetric tridiagonal matrix

$$
\boldsymbol{R}=\left[\begin{array}{ccccc}
0 & \sqrt{1} & 0 & \cdots & 0 \\
\sqrt{1} & 0 & \sqrt{2} & \ddots & \vdots \\
0 & \sqrt{2} & \ddots & \ddots & 0 \\
\vdots & \ddots & \ddots & 0 & \sqrt{N} \\
0 & \cdots & 0 & \sqrt{N} & 0
\end{array}\right]
$$


whose eigenvalues $\mu_{m}$ are related to the desired roots of $H_{N+1}(\xi)$ by the simple formula

$$
\mu_{m}=\sqrt{2} \xi_{m}
$$

for $m=0,1, \ldots, N$.

After having determined the set $\left\{\xi_{m}\right\}$ of Hermite grid points, we are now able to substitute (27) into equation (21) and enforce its satisfaction at the nodes $\xi_{0}, \xi_{1}, \ldots, \xi_{N}$ to reach the algebraic equations

$$
\sum_{n=0}^{N}\left[-\ell_{n}^{\prime \prime}\left(\xi_{m}\right)+2 \xi_{m} \ell_{n}^{\prime}\left(\xi_{m}\right)+\left(q_{m}+1\right) \ell_{n}\left(\xi_{m}\right)\right] y_{n}=\mathcal{E}(\alpha) \sum_{n=0}^{N} \ell_{n}\left(\xi_{m}\right) y_{n}
$$

for $m=0,1, \ldots, N$, leading to the matrix (unsymmetric) representation

$$
\widehat{\mathcal{B}} \boldsymbol{y}=\mathcal{E}(\alpha) \boldsymbol{y}
$$

of the Schrödinger equation, where the general entry $\widehat{B}_{m n}$ of $\widehat{\mathcal{B}}$ is given by

$$
\widehat{B}_{m n}=\widehat{K}_{m n}+\left(q_{m}+1\right) \delta_{m n}, \quad m, n=0,1, \ldots, N
$$

in which $q_{m}:=q\left(\xi_{m}\right)$, and $\widehat{K}_{m n}$ is expressible as

$$
\widehat{K}_{m n}=-d_{m n}^{(2)}+2 \xi_{m} d_{m n}^{(1)}
$$

in terms of the differentiation matrices of section 2. As shown, the kinetic and potential energy terms in the Schrödinger operator are characterized by the general $\widehat{K}_{m n}$ and diagonal matrix elements $\left(q_{m}+1\right) \delta_{m n}$, respectively. Note that an eigenvector $\boldsymbol{y}=\left[y_{0}, y_{1}, \ldots, y_{N}\right]^{\mathrm{T}}$ of (34) with $y_{m}:=y\left(\xi_{m}\right)$, corresponding to a typical eigenvalue $\mathcal{E}$, includes the values of an eigenfunction of the transformed equation (21) evaluated at the nodes.

Weidemann [2] examined the spectral properties of the first and second order differentiation matrices in (36) separately, resulting from the use of the Hermite collocation points. Here, we shall consider the total kinetic energy matrix, which seems to be more reasonable. By inserting (29) into (16) and (19), it is not difficult to write down the elements $\widehat{K}_{m n}$,

$$
\widehat{K}_{m n}= \begin{cases}2 \frac{\left(1-\xi_{m} \xi_{n}+\xi_{m}^{2}\right) \psi_{N+1}^{\prime}\left(\xi_{m}\right)}{\left(\xi_{m}-\xi_{n}\right)^{2} \psi_{N+1}^{\prime}\left(\xi_{n}\right)}-\frac{\psi_{N+1}^{\prime \prime}\left(\xi_{m}\right)}{\left(\xi_{m}-\xi_{n}\right) \psi_{N+1}^{\prime}\left(\xi_{n}\right)} & \text { if } m \neq n \\ \xi_{n} \frac{\psi_{N+1}^{\prime \prime}\left(\xi_{n}\right)}{\psi_{N+1}^{\prime}\left(\xi_{n}\right)}-\frac{\psi_{N+1}^{\prime \prime \prime}\left(\xi_{n}\right)}{3 \psi_{N+1}^{\prime}\left(\xi_{n}\right)} & \text { if } m=n\end{cases}
$$

plainly, which reduce to quite a simpler form

$$
\widehat{K}_{m n}=\frac{2}{3} \begin{cases}\frac{3 \psi_{N}\left(\xi_{m}\right)}{\left(\xi_{m}-\xi_{n}\right)^{2} \psi_{N}\left(\xi_{n}\right)} & \text { if } m \neq n \\ N+\xi_{n}^{2} & \text { if } m=n\end{cases}
$$


after a long but straightforward manipulations, where we have thankfully exploited the functional equations listed in (24)-(26) that exist for the Hermite polynomials.

It is obvious from (37) that $\widehat{K}_{m n} \neq \widehat{K}_{n m}$, and hence the matrix $\widehat{\mathcal{B}}$ is not symmetric. Fortunately, however, it may be symmetrized by a similarity transformation

$$
\mathcal{B}=\boldsymbol{P}^{-1} \widehat{\mathcal{B}} \boldsymbol{P},
$$

whenever $\boldsymbol{P}$ has been chosen as a diagonal matrix of the type

$$
\boldsymbol{P}=\operatorname{diag}\left(\psi_{N, 0}, \psi_{N, 1}, \cdots, \psi_{N, m}, \cdots, \psi_{N, N}\right),
$$

which can immediately be constructed by ordering the last components of each eigenvector of the tridiagonal matrix $\boldsymbol{R}$ in (31), where $\psi_{N, m}:=\psi_{N}\left(\xi_{m}\right)$. Then it follows from (38) that the matrix $\mathcal{B}$ has entries $B_{m n}$ of the form

$$
B_{m n}=K_{m n}+\left(q_{m}+1\right) \delta_{m n}, \quad m, n=0,1, \ldots, N
$$

and it is indeed symmetric since

$$
K_{m n}=\frac{2}{3} \begin{cases}\frac{3}{\left(\xi_{m}-\xi_{n}\right)^{2}} & \text { if } m \neq n \\ N+\xi_{n}^{2} & \text { if } m=n\end{cases}
$$

with $K_{m n}=K_{n m}$. Thus we can replace (34) with the symmetric eigenvalue problem

$$
\mathcal{B} \boldsymbol{u}=\mathcal{E}(\alpha) \boldsymbol{u}
$$

on recalling the fact that $\mathcal{B}$ shares the same spectrum with $\widehat{\mathcal{B}}$. Furthermore, since

$$
\boldsymbol{P}^{-1} \widehat{\mathcal{B}} \boldsymbol{P} \boldsymbol{u}=\mathcal{E} \boldsymbol{u} \Rightarrow \widehat{\mathcal{B}}[\boldsymbol{P u}]=\mathcal{E}[\boldsymbol{P u}]
$$

we infer that an eigenvector $\boldsymbol{y}$ of (34) is given by

$$
\boldsymbol{y}=\boldsymbol{P u}
$$

in terms of an eigenvector $\boldsymbol{u}=\left[u_{0}, u_{1}, \ldots, u_{N}\right]^{\mathrm{T}}$ of the symmetric matrix $\mathcal{B}$. On back substitution of $\boldsymbol{u}$ into (44), it is seen from (20) that a typical member $u_{m}$ of an eigenvector stands for

$$
u_{m}=\psi_{N, m}^{-1} y_{m}=\left[\psi_{N}\left(\xi_{m}\right)\right]^{-1} \mathrm{e}^{\xi_{m}^{2} / 2} \Psi\left(\xi_{m}\right)
$$

which determines the original wavefunction $\Psi(\xi)$,

$$
\Psi\left(\xi_{m}\right)=u_{m} \psi_{N}\left(\xi_{m}\right) \mathrm{e}^{-\xi_{m}^{2} / 2}
$$

at a grid point. 
On the other hand, if the energy spectrum of the Schrödinger operator $\mathcal{H}$ in (1) will be primarily of interest, all we need is to compute the eigenvalues $\mathcal{E}$ of (42) and then use the passage formula $E=\alpha^{2} \mathcal{E}(\alpha)$ designated by (4). It is worth mentioning once again that the symmetric matrix elements $B_{m n}$ in (40) entail only the knowledge of zeros $\xi_{m}$ of $H_{N+1}(\xi)=0$ and the values of a prescribed potential function at the nodes.

\section{Numerical results and discussion}

A comprehensive test of the numerical procedure of section 3 has been performed for each quantum mechanical system considered in PI. However, we render here two cases which reflect successfully the overall principal features of the HPM. To be specific, exemplary computations for a Morse potential (MP) of the form

$$
V(x)=\left(\mathrm{e}^{-\gamma x}-1\right)^{2}, \quad 0<\gamma<2
$$

and a symmetric double well potential (SDWP)

$$
V(x)=v_{4}\left(x^{2}-\frac{1}{2} v_{4}^{-1}\right)^{2}, \quad v_{4}>0
$$

are displayed in tables 1 and 2, respectively. The analytically solvable MP in (47) has a finite number of discrete spectral points lying between $0<E_{n}<1$ and a continuous spectrum for all $E \geqslant 1$ [7]. The discrete eigenvalues are expressible as

Table 1

Comparison of the convergence rates of Hermite-Weber basis in the Rayleigh-Ritz and pseudospec-

\begin{tabular}{|c|c|c|}
\hline$N$ & $E_{0}$ in $\mathrm{HPM}$ & $E_{0}$ in Rayleigh-Ritz \\
\hline 10 & 0.01564620 & 0.0156463 \\
\hline 15 & 0.01564627822 & 0.01564627828 \\
\hline 20 & 0.015646278241 & 0.015646278243 \\
\hline 25 & 0.015646278240444 & 0.01564627824045 \\
\hline 30 & 0.015646278240442165 & 0.015646278240442159 \\
\hline 35 & 0.01564627824044215769 & 0.01564627824044215771 \\
\hline 40 & 0.01564627824044215770067 & 0.0156462782404421577012 \\
\hline 45 & 0.015646278240442157700942 & 0.01564627824044215770095 \\
\hline 50 & 0.01564627824044215770094548 & 0.0156462782404421577009458 \\
\hline 55 & 0.0156462782404421577009455012 & 0.015646278240442157700945502 \\
\hline 58 & 0.0156462782404421577009455014 & 0.0156462782404421577009455014 \\
\hline 60 & 0.01564627824044215770094550135 & 0.01564627824044215770094550135 \\
\hline 61 & 0.01564627824044215770094550135 & 0.01564627824044215770094550135 \\
\hline
\end{tabular}
tral methods. 
Table 2

Nearly degenerate states of the SDWP in (48) for $v_{4}=0.01$.

\begin{tabular}{rccccccc}
\hline$n$ & $N_{\mathrm{RR}}$ & $N_{\mathrm{HPM}}$ & $E_{n}$ & & $\alpha_{\mathrm{opt}}$ \\
\hline 0 & 52 & 104 & 1.40404860529770688242570757082 & 1.0 \\
1 & 52 & 104 & 1.40404860529770688260256628056 & 1.0 \\
2 & 54 & 108 & 4.17019360599931012783387507130 & 1.0 \\
3 & 54 & 108 & 4.17019360599931021961329119873 & 1.0 \\
4 & 56 & 112 & 6.87008883371402461217231516849 & 1.0 \\
5 & 56 & 112 & 6.87008883371404680242599568189 & 1.0 \\
6 & 58 & 116 & 9.48957838718787005519441835655 & 1.0 \\
7 & 58 & 116 & 9.498578387191178212320856961 & 14 & 1.0 \\
8 & 59 & 118 & 12.049309486334092592332880171 & 6 & 1.1 \\
9 & 59 & 118 & 12.049309486673006847573312477 & 9 & 1.1 \\
10 & 60 & 120 & 14.514205022981239103429421443 & 9 & 1.1 \\
11 & 60 & 120 & 14.514205048121017338991612415 & 8 & 1.1 \\
\hline
\end{tabular}

$$
E_{n}=\left(n+\frac{1}{2}\right) \gamma\left[2-\left(n+\frac{1}{2}\right) \gamma\right], \quad n=0,1, \ldots, \llbracket \gamma^{-1}-\frac{1}{2} \rrbracket
$$

where $\llbracket a \rrbracket$ denotes the integer part of a real number $a$.

In table 1, numerical results for the ground state eigenvalue of the MP are presented as a function of the truncation size $N$, where the potential and optimization parameters are $\gamma=0.005 \pi$ and $\alpha=0.1$, respectively. As $N$ increases, it is shown that the HPM generates a sequence of approximations oscillating about the exact analytical result

$$
E_{0}=0.015646278240442157700945501348
$$

calculated from (49). Whereas the Rayleigh-Ritz method provides always upper bounds converging to the true limit from above as it is based on a variational principle. Nevertheless, both algorithms reach the target at the same truncation order of $\mathrm{N}=60$.

The SDWP in (48) is an example of a quartic polynomial oscillator in which the two wells are symmetrically located about the origin. The crucial aspect of the spectrum of a SDWP is that the low-lying state energies are very closely bunched in pairs if the wells are sufficiently separated, which occurs for weak couplings when $v_{4} \ll 1$. In this case, the determination of the gaps between the nearly degenerate energy levels turns out to be more substantial and requires indeed a high precision numerical algorithm [8].

We exhibit such states of a SDWP in table 2, where $N_{\mathrm{RR}}$ and $N_{\mathrm{HPM}}$ indicate matrix sizes of the Rayleigh-Ritz and pseudospectral schemes, respectively, at which the desired accuracy is obtained. It is shown that $N_{\mathrm{HPM}}=2 N_{\mathrm{RR}}$. Despite this doubling in the matrix sizes, one should not conclude that the rate of convergence of the HPM is slower. As a matter of fact, the energy spectrum of the 
SDWP can be decomposed into two subsets consisting of even and odd eigenlevels, separately, owing to its reflection symmetry, $V(x)=V(-x)$. In PI, the Rayleigh-Ritz trial function in (23) had been modified simply by replacing $\left\{\psi_{n}\right\}$ with the bases $\left\{\psi_{2 n}\right\}$ and $\left\{\psi_{2 n+1}\right\}$ in turn, so as to deal with the even and odd parity wavefunctions, respectively. Therefore, in order to halve the dimensions of the resulting matrices in the HPM, the reflection symmetric structure of the Schrödinger operator (1) for a potential with $V(x)=V(-x)$ has to be taken into account. Unfortunately, however, an immediate rearrangement of the polynomial interpolant in (27) is not possible, and our research effort to this end is still continuing.

In all calculations, we observe that there is virtually no discrepancy in the optimal $\alpha_{\text {opt }}$ values. This is due to the fact that the parameter $\alpha$ is affected to a large extent by the asymptotic form of the wavefunctions. It is clear from (20) that, we have already assigned the asymptotic structures of both Rayleigh-Ritz and pseudospectral trial solutions to be $\mathrm{e}^{-\xi^{2} / 2}$ as $\xi \rightarrow \infty$.

In the Rayleigh-Ritz method, the scaled Hamiltonian in (3) were represented by the variational matrix $\mathcal{A}:=\left[\mathcal{A}_{m n}\right]$ with

$$
\mathcal{A}_{m n}=(2 n+1) \delta_{m n}+Q_{m n}, \quad m, n=0,1, \ldots, N
$$

in which the diagonal $(2 n+1) \delta_{m n}$ and the general $Q_{m n}$,

$$
Q_{m n}=\int_{-\infty}^{\infty} q(\xi) \phi_{m}(\xi) \phi_{n}(\xi) \mathrm{d} \xi
$$

matrix elements are the descriptions of the kinetic and potential energies, respectively. Recall, from (35) or (40), that the situation is completely reversed in the HPM. Theoretically, as $N \rightarrow \infty$, it is natural to expect that the infinitedimensional matrices $\mathcal{A}$ and $\mathcal{B}$ (or $\widehat{\mathcal{B}}$ ) would have become similar. However, the fact confirmed evidently by the numerical experiments that the truncated versions of $\mathcal{A}$ and $\mathcal{B}$ yield the same accuracy for the eigenvalues of the Schrödinger operator at the same finite matrix orders $N$ is really surprising.

Finally, it seems that the symmetrization process in (38) is very closely related to the so-called quadrature discretization method (see, for example, [9] and the references therein). The derivation of explicit analytical connections is presently an ongoing study as well, and the results will be reported in due course.

\section{Acknowledgment}

This research was supported by a grant from TUBITAK, the Scientific and Technical Research Council of Turkey. 


\section{References}

[1] H. Taşeli and M.B. Erseçen, J. Math. Chem. 34 (2003) 177.

[2] J.A.C. Weideman, Numer. Math. 61 (1992) 409.

[3] T. Tang, SIAM J. Sci. Comput. 14 (1993) 594.

[4] D. Funaro, Polynomial Approximations of Differential Equations (Springer-Verlag, Berlin, 1992).

[5] F.B. Hildebrand, Introduction to Numerical Analysis (McGraw-Hill, New York, 1956).

[6] B.D. Welfert, SIAM J. Numer. Anal. 34 (1997) 1640.

[7] H. Taşeli, J. Comput. Appl. Math. 115 (2000) 535.

[8] K. Banerjee and S.P. Bhatnagar, Phys. Rev. D 18 (1978) 4767.

[9] K. Leung, B.D. Shizgal and H. Chen, J. Math. Chem. 24 (1998) 291. 